\title{
AC 2012-3063: USING A PAIR OF IPODS TO MEASURE ANGLE OF TWIST IN A TORSION EXPERIMENT
}

Dr. Surendra K. Gupta, Rochester Institute of Technology

"Vinnie" Gupta is a professor of mechanical engineering, and a member of the graduate faculty of materials science and engineering at the Rochester Institute of Technology (Rochester, N.Y.). He is a recipient of the 2000 Eisenhart Award for Excellence in Teaching. At RIT, he teaches undergraduate and graduate courses in applied mechanics, computational techniques, and materials science.

\section{Mr. Steven John Kosciol, Rochester Institute of Technology}

Steven John Kosciol is Lab Manager of the Mechanical Engineering Machine Shop. He teaches the lab section of the course "Manufacturing Process." This introduces the students to the machine shop environment and hands-on engineering. 


\section{Using a pair of iPods to Measure Angle of Twist in a Torsion Experiment}

\section{Introduction}

Every mechanical engineering undergraduate student at our university must take a sophomore level one quarter-credit hour course titled "Mechanics of Materials Lab". A four quarter-credit hour Mechanics of Materials course is a co-requisite to this laboratory course. One of the five experiments in the laboratory course focuses on the study of elastic and plastic deformation of a circular cross-section metallic rod in torsion.

The objectives of the torsion experiment include determination of shear modulus of elasticity "G", shear proportional limit " $\tau_{\mathrm{p}}$ ", and the mode of failure of the material. In the torsion test, a torque " $T$ " is applied to one end of a circular cross-section metallic rod while the other end is held fixed in a stationary grip. We use a bench-mounted Tinius Olsen 10,000 in-lb Torsion Tester shown in Figure 1. The tester has a variable speed drive electromechanical loading system with manual controls and LED digital display. We have developed a LabView interface to acquire the torque and grip angular position data using the RS-232 port.

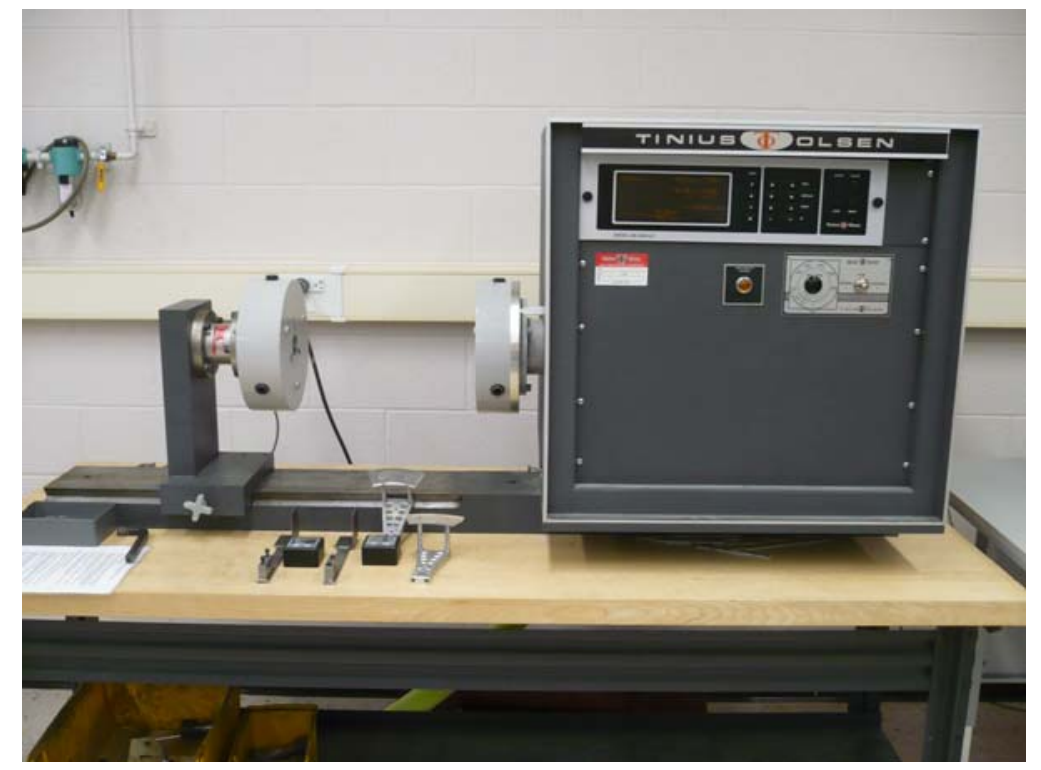

Figure 1: Tinius Olsen 10,000 in-lb Torsion Tester

The shear strain " $\gamma$ " on the surface of the rod is determined by measuring the relative angle of twist " $\phi_{\mathrm{G}}$ " over a gage length " $\mathrm{L}_{\mathrm{G}}$ ". The shear strain $\gamma=\mathrm{c} * \phi_{\mathrm{G}} / \mathrm{L}_{\mathrm{G}}$ where "c" is the radius of cross-section and $\phi_{\mathrm{G}}$ is in radians. With shear stress " $\tau$ " linearly proportional to shear strain $\gamma$ for $\tau \leq \tau_{\mathrm{p}}$, the shear stress at the surface is $\tau=2 \mathrm{~T} /\left(\pi \mathrm{c}^{3}\right)$. The shear modulus $\mathrm{G}$ is then determined as the slope of the line fitted to $\tau$ versus $\gamma$ data. 
Until a few years ago, the relative angle of twist $\phi_{\mathrm{G}}$ was measured using a troptometer shown in Figure 2 that was designed and fabricated in-house more than thirty years ago. It consists of two halves - each consisting of a $45^{\circ}$ plastic protractor attached to a Y-shaped aluminum plate that is clamped onto the specimen using two hexagonal head screws.

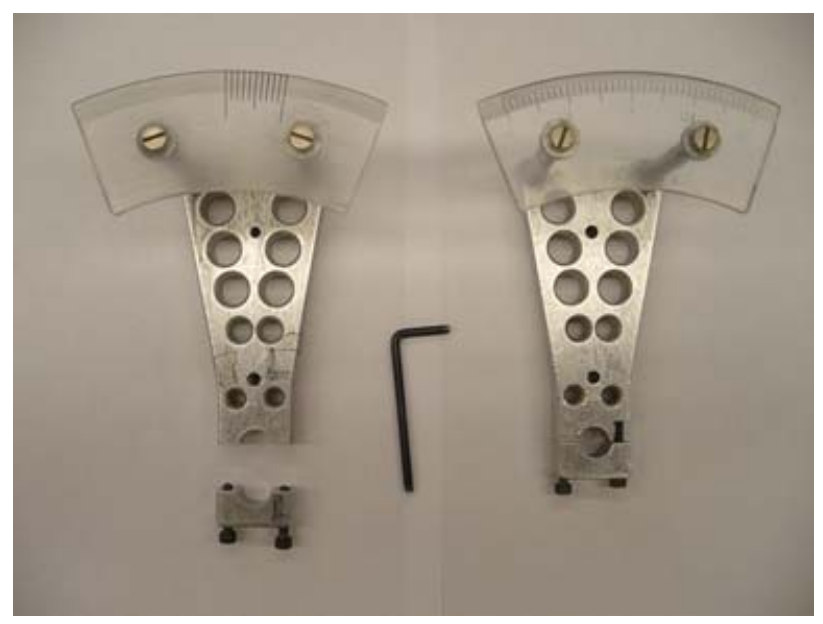

Figure 2a: Older Troptometer

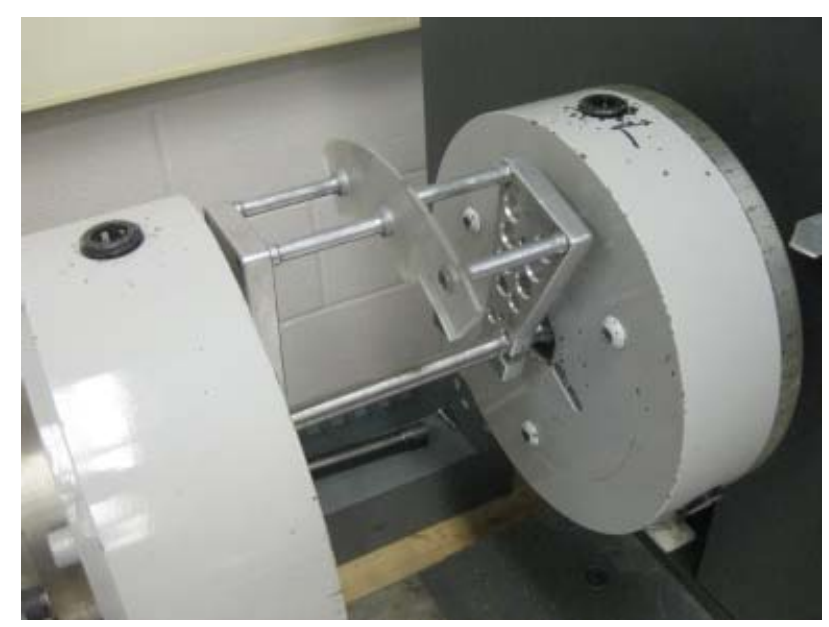

Figure 2b: Mounted on a specimen in Torsion Tester

Students experienced three difficulties in using this troptometer: (i) keeping it aligned while mounting it on the specimen, (ii) reading $\phi_{\mathrm{G}}$ from the protractor scale, and (iii) taking it off when the specimen begins to deform plastically.

\section{Digital Inclinometers}

Two years ago, we designed and fabricated a pair of specimen clamps shown in Figure $3 \mathrm{a}$ to which a pair of commercially available digital inclinometers could be magnetically attached. The clamps are made of low carbon steel that can be easily machined, formed and welded.

It was quite easy to mount each clamp onto a specimen. The specimen with the clamps was then mounted in the torsion tester grips. A digital inclinometer was magnetically attached to each clamp as shown in Figure 3b, powered on, and initialized to display relative zero degree orientation. This eliminated difficulty (i) experienced with the troptometer.

As the applied torque $\mathrm{T}$ was increased on the specimen, the display on the inclinometer on the right " $\phi_{R}$ ", and on the left " $\phi_{\mathrm{L}}$ " was read simultaneously. The relative angle of twist $\phi_{\mathrm{G}}=\phi_{\mathrm{R}}-\phi_{\mathrm{L}}$ was thus easily determined. This eliminated difficulty (ii) experienced with the troptometer. In practice, $\phi_{\mathrm{R}}$ readings were recorded at $0.5^{\circ}$ intervals up to $10^{\circ}$, and at $1^{\circ}$ intervals up to $20^{\circ}$ thereafter. 


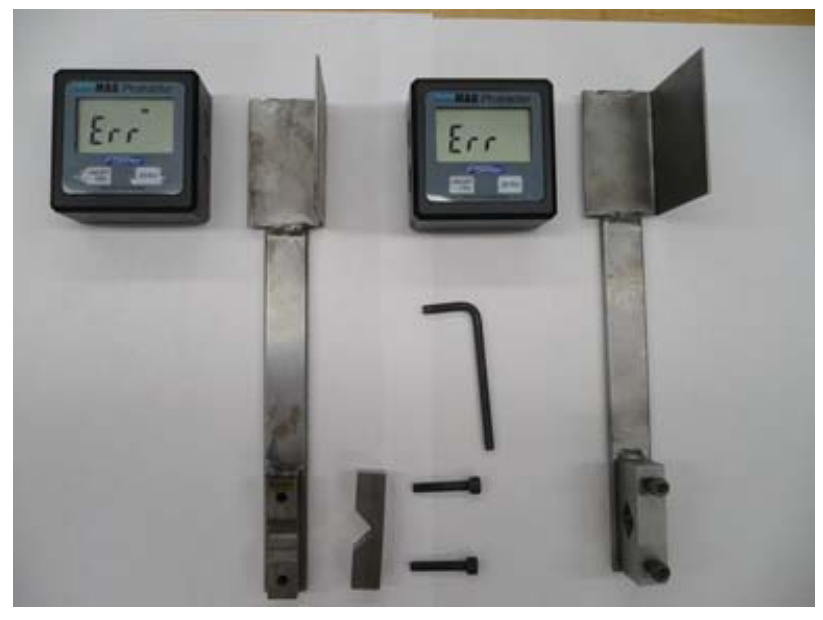

Figure 3a: Inclinometers and Specimen Clamps

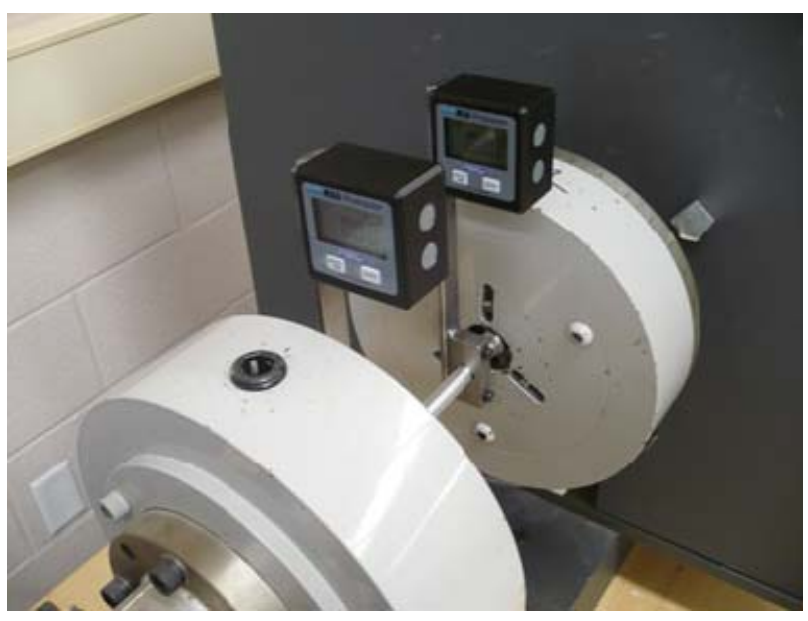

Figure 3b: Attached to specimen clamps in Torsion Tester

When $\phi_{\mathrm{R}}$ exceeded $20^{\circ}$, the two inclinometers were detached from the clamps, and kept aside. The clamps did not need to be removed from the specimen. This eliminated difficulty (iii) experienced with the troptometer. Since each inclinometer was detached magnetically, the removal operation was very safe. The torsion test was continued until the specimen failed so that students could examine the fracture surface, and determine whether the failure mode was ductile or brittle.

Thus, using commercially available digital inclinometers attached to specimen clamps shown in Figure 3 eliminated all three difficulties encountered with a troptometer ${ }^{1}$.

Torsion tests were conducted on 2024-T351 aluminum alloy and 1018 cold rolled steel specimens using both $\phi_{\mathrm{G}}$ measuring techniques. Nominal shoulder to shoulder distance and diameter of each specimen was $71 / 8$ " and $1 / 2$ " respectively as prescribed in ASTM E-143 standard. Both techniques to measure the relative angle of twist yielded comparable values for shear modulus. Even though the line fits were excellent in all cases, we found that the correlation coefficient of line fit on data from inclinometers was always less than that from troptometer on a corresponding specimen. The marginally poorer correlation coefficient obtained with inclinometers was attributed to their sluggish response in measuring and displaying the angular tilts. The inclinometer display changed by as much as $0.15^{\circ}$ often. The inclinometer determines the angular tilt by measuring the capacitance of a liquid column whose height changes with tilt. The inclinometer's electronic equilibration and response is quite sluggish. These inclinometers are designed for applications where either the tilt angle is either static or tilt angle changes due to vibrations need to be minimized by damping.

\section{Using iPod Touch}

Last year, we designed and fabricated another pair of metallic clamps and plastic holders shown in Figure 4 in which a pair of iPod Touch devices can be inserted. Initially, the clamps and the rods were made of low carbon steel that can be easily machined and threaded. The ABS plastic 
holders were fabricated by rapid prototyping using a Dimension 3D Printer. Tilt angle was measured on each iPod by a $\$ 0.99$ "app" called "Clinometer" downloaded from the iTunes App Store $^{2}$. The app uses iPod's built-in accelerometer which measures the acceleration of the device relative to free fall $(=1 \mathrm{~g})$ in three different axes: $\mathrm{X}, \mathrm{Y}$ and $\mathrm{Z}$. Holding the device vertically in portrait mode, $\mathrm{X}$-axis is left to right, $\mathrm{Y}$-axis is bottom to top, and $\mathrm{Z}$-axis is back to front. The accelerometer $^{3}$ has a measurement range of $\pm 2.3 \mathrm{~g}$ with a resolution of $0.018 \mathrm{~g}$, and allows detection of the orientation of the device. The app developers claim a precision of $\pm 0.1^{\circ}$ in tilt angle measurement. In Figure 5, if the display on the right iPod is " $\phi_{\mathrm{R}}$ ", and on the left iPod is " $\phi_{\mathrm{L}}$ ", then relative angle of twist $\phi_{\mathrm{G}}=\phi_{\mathrm{R}}-\phi_{\mathrm{L}}$.

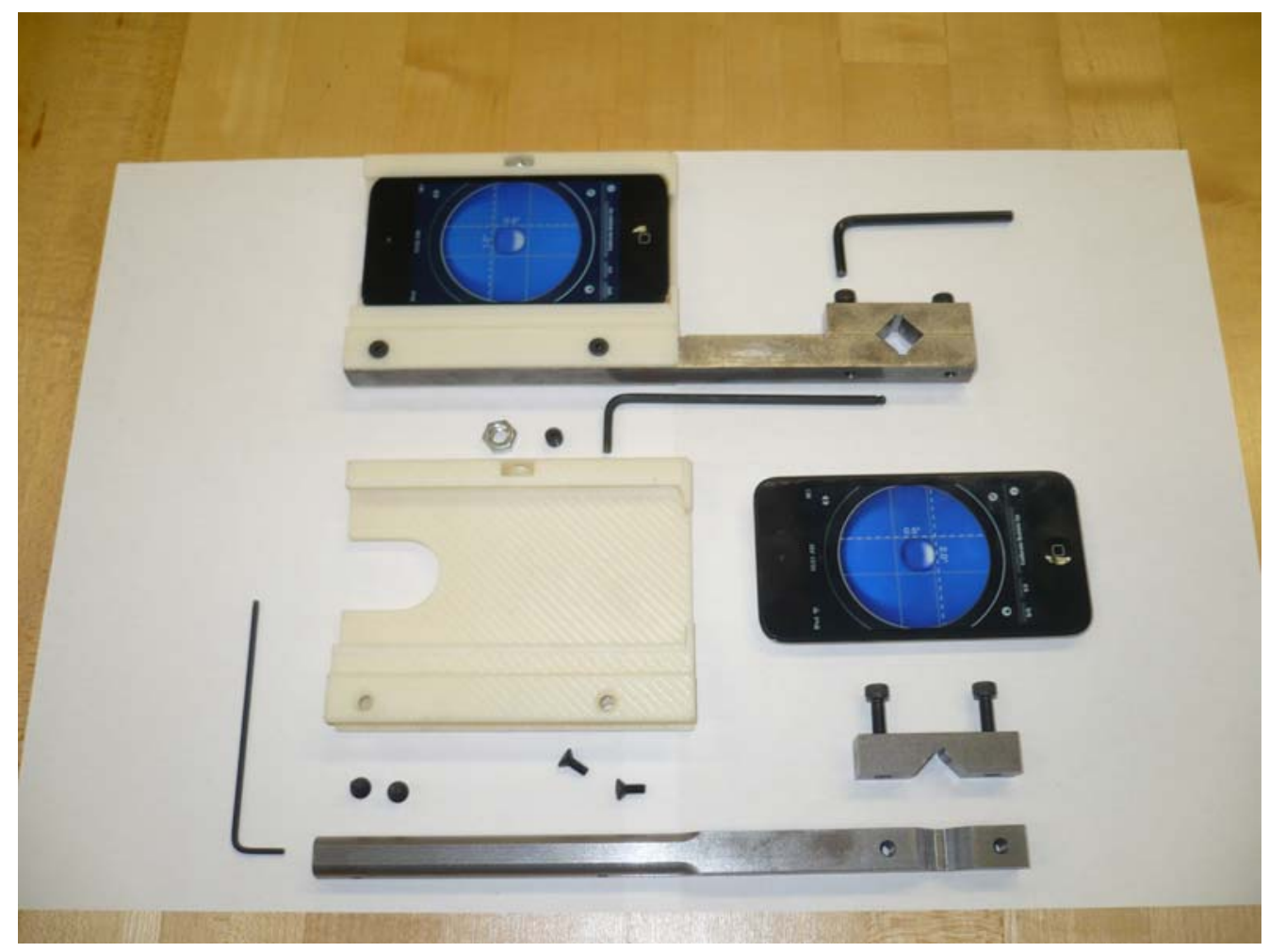

Figure 4: Steel Clamps with ABS Plastic iPod Holders and a pair of iPods

When torsion tests were conducted on 1018 steel specimens, even though the iPods were held quite snuggly in the plastic holders, it was difficult to read the digital angular displays due to significant vibrations. The variable speed drive vibrations of the torsion tester were being transmitted through the grips to the steel specimen followed by steel clamps to steel rods to which iPod holders were attached. When the torsion tests were conducted on 2024 aluminum alloy specimens, vibrations experienced by iPods were negligible and tilt angles could easily be read at all torque values. We concluded that the aluminum specimens were over-damping the vibrations at the torsion tester grips.

In the next iteration, we fabricated the clamps and rods from 6061 aluminum alloy. To reinforce the threads, Heli-Coil inserts were used in the tapped holes of clamps. To increase their strength, wear resistance, and durability, the clamps and rods were anodized. With iPod holders shown in Figure 5, no vibrations were observed in torsion testing of either steel or aluminum specimens. 


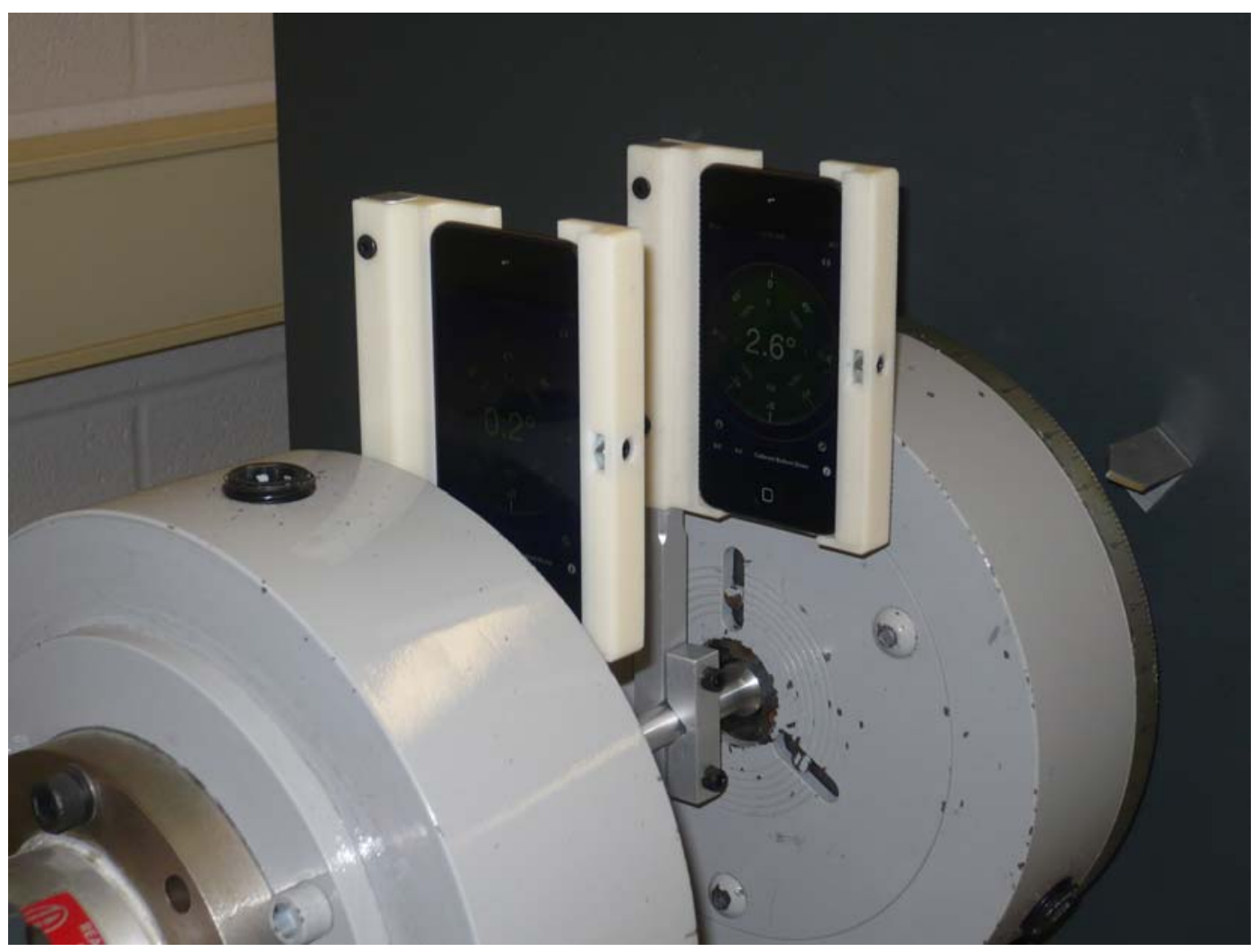

Figure 5: Aluminum Clamps with ABS Plastic iPod Holders mounted on an aluminum specimen

\section{Experimental Results and Discussion}

In measurements reported below, we kept the distance $\mathrm{L}_{\mathrm{G}}$ between the two clamps to be approximately $4 \frac{1}{2}$ " to achieve comparable precision in $\phi_{\mathrm{G}}$ measurements by both inclinometers and iPods. Figure 6 shows a plot of shear stress versus shear strain data points in the elastic range with least-square line fits to determine the shear modulus of 2024-T351 aluminum alloy specimens. Since the inclinometer display changes by as much as $0.15^{\circ}$ often, the horizontal error bars for inclinometer data reflect this $0.15^{\circ}$ uncertainty whereas the iPod data error bars reflect the Clinometer display resolution of $0.1^{\circ}$. Table 1 below summarizes the results from least-squares line fitting for both steel and aluminum alloy specimens:

Table 1: Results from Experimental Measurements

\begin{tabular}{|l|c|c|c|c|}
\hline & \multicolumn{2}{|c|}{ 2024-T351 Aluminum Alloy } & \multicolumn{2}{c|}{ 1018 Cold Rolled Steel } \\
\cline { 2 - 5 } & Inclinometer & iPod & Inclinometer & iPod \\
\hline Shear Modulus G (Msi) & 3.96 & 4.15 & 12.37 & 12.32 \\
\hline Correlation Coefficient $\mathbf{r}^{\mathbf{2}}$ & 0.9996 & 0.9918 & 0.9838 & 0.9937 \\
\hline
\end{tabular}




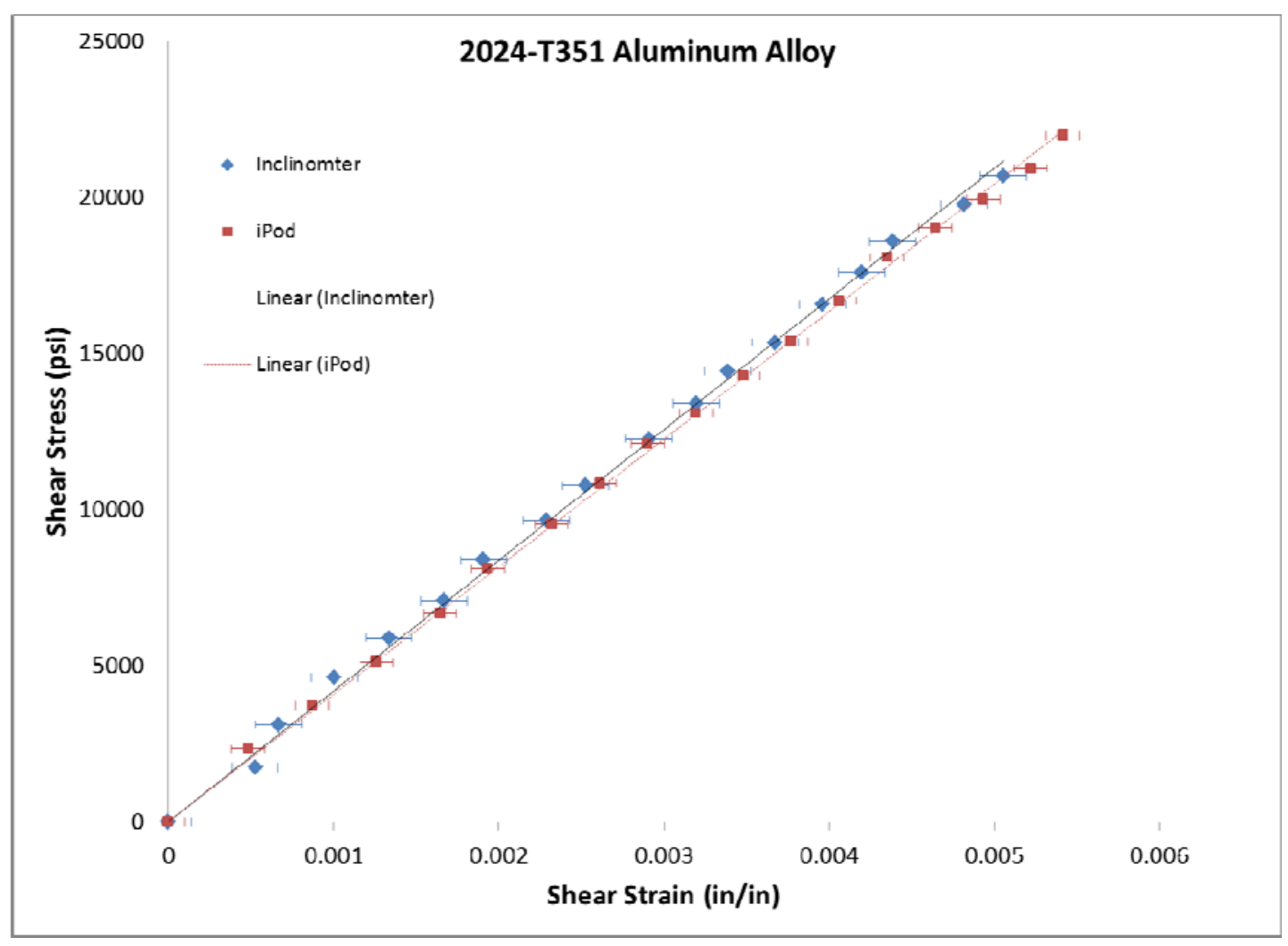

\section{Figure 6: Plot of Shear Stress versus Shear Strain}

From results of a previous laboratory experiment on tensile testing conducted to determine the elastic modulus and Poisson's Ratio of both these materials, we can compute the shear modulus $\mathrm{G}$ to be 4.13 Msi and 11.78 Msi for 2024-T351 Al-Cu and 1018-CRS respectively. Table 1 results are within $5 \%$ of these values.

\section{Conclusions}

1. Students encountered three difficulties in using the older troptometer: (i) keeping it aligned while mounting it on a specimen, (ii) reading its protractor scale, and (iii) removing it from the specimen during plastic deformation of the specimen.

2. Using commercially available digital inclinometers attached to specimen clamps eliminated all three difficulties identified in item \#1. Both techniques to measure the relative angle of twist yielded comparable values for shear modulus and linear correlation coefficient. The marginally poorer correlation coefficient obtained with inclinometers was attributed to their sluggish response.

3. When an inclinometer was replaced by a Clinometer enabled iPod, the torsion tester vibrations were transmitted via the steel specimen to the steel clamp and rod to the iPod holder making it impossible to read the tilt angle on the Clinometer display. However, an 
aluminum alloy specimen over-damped the torsion tester vibrations, and made it possible to read the tilt angle display.

4. A redesigned aluminum alloy clamp and rod over-damped the torsion tester vibrations, and made it possible to read the Clinometer display for both steel and aluminum specimens.

5. The tapped holes in clamps were reinforced using Heli-Coils. To increase their strength, wear resistance and durability, the aluminum clamps and rods were anodized.

6. The "Clinometer" iPod tilt angle measuring technique with anodized aluminum clamp \& rod, and plastic iPod holder yields experimental values of shear modulus in torsion testing that are comparable or better than other measurements.

\section{Acknowledgements}

The authors gratefully acknowledge the help provided by: (i) Devin Hamilton and John Bonzo in designing and fabricating the iPod holders by rapid prototyping using a Dimension 3D Printer; (ii) Jan Maneti in fabricating the metallic clamps and rods; and (iii) Kelsey McConnaghy, Jared Dodge, Bradley Ling, and Markus Holzenkamp in conducting the torsion tests during the preparation of this manuscript.

\section{References}

1. S. K. Gupta and S. Kosciol, Measuring Angle of Twist in a Torsion Experiment, Proceedings of the ASEE Annual Conference in Vancouver, BC (June 2011). AC 2011-77.

2. Plaincode Software Solutions, Clinometer on iPhone / iPod Touch, accessed on March 8, 2012. HTML.

3. STMicroelectronics, Datasheet on LIS302DL 3-axis MEMS Motion Sensor, accessed on March 8, 2012. PDF. 